\title{
Discrete Morse Theory for free chain complexes
}

\section{Théorie de Morse pour des complexes de chaines libres}

\author{
Dmitry N. Kozlov \\ Eidgenössische Technische Hochschule, Zürich, Switzerland
}

\begin{abstract}
We extend the combinatorial Morse complex construction to the arbitrary free chain complexes, and give a short, self-contained, and elementary proof of the quasi-isomorphism between the original chain complex and its Morse complex.

Even stronger, the main result states that, if $C_{*}$ is a free chain complex, and $\mathcal{M}$ an acyclic matching, then $C_{*}=C_{*}^{\mathcal{M}} \oplus T_{*}$, where $C_{*}^{\mathcal{M}}$ is the Morse complex generated by the critical elements, and $T_{*}$ is an acyclic complex. To cite this article: D.N. Kozlov, C. R. Acad. Sci. Paris, Ser. I ??? (2005).

\section{Résumé}

On étend la construction du complex de Morse combinatoire aux complexes de chaines libres générals, et on donne une demonstration brève et élémentaire du quasi-isomorphisme entre le complex de chaines original et son complex de Morse.

Plus profondement, le résultat principal dit que, si $C_{*}$ est un complex de chaines libres, et $\mathcal{M}$ est une correspondence acyclique, puis $C_{*}=C_{*}^{\mathcal{M}} \oplus T_{*}$, et $C_{*}^{\mathcal{M}}$ est le complexe de Morse généré par les éléments critiques, et $T_{*}$ est un complex acyclique. Pour citer cet article : D.N. Kozlov, C. R. Acad. Sci. Paris, Ser. I??? (2005).
\end{abstract}

Version française abrégée

La théorie de Morse discrète a été introduite par Forman [1], et elle a prouvé d'être profitable pour des calculations en combinatoire topologique. Il a été demontré en [1, Theorem 8.2] que, donné une fonction de Morse discrète [1, Definition 2.1] sur un complex CW fini $K$, le complex de chaines cellulair $C_{*}(K ; \mathbb{Z})$ est quasi-isomorphique au complex de Morse combinatoire associé.

\footnotetext{
Email address: dkozlov@inf.ethz.ch (Dmitry N. Kozlov).

URL: http ://www.ti.inf.ethz.ch/people/kozlov.html (Dmitry N. Kozlov).

1 Research supported by Swiss National Science Foundation Grant PP002-102738/1
} 
Dans cet article, on étend cette construction au cas de complexes de chaines libres génerals. On présente une demonstration independente et simple dans cette géńeralité, en particulier, on arrive a une demonstration nouvelle et élémentaire des résultats de Forman. Sur un niveau plus élévé, on peut regarder notre demonstration comme un analogue algebrique des arguments présentés en [2, Theorem 3.2].

Soit $\mathcal{R}$ un anneau commutative général avec un element neutre. On dit qu' un complex de chaines $C_{*}$ aux $\mathcal{R}$-modules . . $\stackrel{\partial_{n+2}}{\longrightarrow} C_{n+1} \stackrel{\partial_{n+1}}{\longrightarrow} C_{n} \stackrel{\partial_{n}}{\longrightarrow} C_{n-1} \stackrel{\partial_{n-1}}{\longrightarrow} \ldots$, est libre si chaque $C_{n}$ est un $\mathcal{R}$-module libre généré au fini. Si les indices sont clairs, on ecrit $\partial$ au lieux de $\partial_{n}$ On demande que $C_{*}$ est borné a droite.

Soupçonné qu'on a choisit un base (un ensemble de générateurs libres) $\Omega_{n}$ pour chaque $C_{n}$. En ce cas, on dit qu'on a choisit un base $\Omega=\bigcup_{n} \Omega_{n}$ pour $C_{*}$, et on ecrit $\left(C_{*}, \Omega\right)$ pour un complex de chaines avec un base. Un complex de chaines libre avec un base est le charactère principal de cet article.

Définition 0.1 Soit $\left(C_{*}, \Omega\right)$ un complex de chaines libre avec un base.

(1) Une correspondence partielle $\mathcal{M} \subseteq \Omega \times \Omega$ sur $\left(C_{*}, \Omega\right)$ est une correspondence partielle sur le diagramme Hasse de $P\left(C_{*}, \Omega\right)$, tel que, si $b \succ a$, et $b$ et a sont en correspondence, i.e. si $(a, b) \in M$, donc $w(b \succ a)$ est invertible.

(2) Une correspondence partielle sur $\left(C_{*}, \Omega\right)$ est acyclique, s'il n'y a pas de cycle

$$
d\left(b_{1}\right) \prec b_{1} \succ d\left(b_{2}\right) \prec b_{2} \succ d\left(b_{3}\right) \prec \ldots \succ d\left(b_{n}\right) \prec b_{n} \succ d\left(b_{1}\right)
$$

avec $n \geq 2$, est tous $b_{i} \in \mathcal{U}(\Omega)$ différents.

Définition 0.2 Soit $\left(C_{*}, \Omega\right)$ un complex de chaines libre avec un base, et soit $\mathcal{M}$ une correspondence acyclique. Le complex de Morse ... $\stackrel{\partial_{n+2}^{\mathcal{M}}}{\longrightarrow} C_{n+1}^{\mathcal{M}} \stackrel{\partial_{n+1}^{\mathcal{M}}}{\longrightarrow} C_{n}^{\mathcal{M}} \stackrel{\partial_{n}^{\mathcal{M}}}{\longrightarrow} C_{n-1}^{\mathcal{M}} \stackrel{\partial_{n-1}^{\mathcal{M}}}{\longrightarrow} \ldots$, est defini comme suit. Le $\mathcal{R}$-module $C_{n}^{\mathcal{M}}$ est librement généré par les elements de $\mathcal{C}_{n}(\Omega)$. L'operateur de borne est defini par $\partial_{n}^{\mathcal{M}}(s)=\sum_{p} w(p) \cdot p_{\bullet}$, pour $s \in \mathcal{C}_{n}(\Omega)$, quand la somme est prise sur tous les chemins alternés $p$ qui satisfaient $p^{\bullet}=s$.

Le complex de chaines ... $\rightarrow 0 \longrightarrow \mathcal{R} \stackrel{\text { id }}{\longrightarrow} \mathcal{R} \longrightarrow 0 \longrightarrow \ldots$, dans lequels les seules modules non-trivials se trouvent en dimension $d$ et $d-1$, on l'appelle un complex de chaines atomique, et on ecrit Atom $(d)$.

Le résultat principal de cet article est comme suit :

Théorème 0.3 Soupçonne qu'on a un complex de chaines libre avec un base $\left(C_{*}, \Omega\right)$, et une correspondence acyclique $\mathcal{M}$. Puis, $C_{*}$ se decompose en un somme direct de complexes de chaines $C_{*}^{\mathcal{M}} \oplus T_{*}$, et $T_{*} \simeq \bigoplus_{(a, b) \in \mathcal{M}} \operatorname{Atom}(\operatorname{dim} b)$.

\section{Acyclic matchings on chain complexes and the Morse complex.}

Discrete Morse theory was introduced by Forman, see [1], and it proved to be useful in various computations in topological combinatorics. It was shown, [1, Theorem 8.2], that, given a discrete Morse function, $\left[1\right.$, Definition 2.1], on a finite CW complex $K$, the cellular chain complex $C_{*}(K ; \mathbb{Z})$ is quasi-isomorphic to the associated combinatorial Morse complex.

In this paper, we extend this construction to the case of arbitrary free chain complexes. We give an independent, simple, and self-contained proof in this generality, in particular furnishing a new elementary and short derivation of the Forman's result. On a higher level, our proof can be viewed as an algebraic analog of the argument given in [2, Theorem 3.2].

Let $\mathcal{R}$ be an arbitrary commutative ring with a unit. We say that a chain complex $C_{*}$ consisting of $\mathcal{R}$ modules . . $\stackrel{\partial_{n+2}}{\longrightarrow} C_{n+1} \stackrel{\partial_{n+1}}{\longrightarrow} C_{n} \stackrel{\partial_{n}}{\longrightarrow} C_{n-1} \stackrel{\partial_{n-1}}{\longrightarrow} \ldots$, is free if each $C_{n}$ is a finitely generated free $\mathcal{R}$-module. When the indexing is clear, we simply write $\partial$ instead of $\partial_{n}$. We require $C_{*}$ to be bounded on the right. 
Assume that we have chosen a basis (i.e. a set of free generators) $\Omega_{n}$ for each $C_{n}$. In this case we say that we have chosen a basis $\Omega=\bigcup_{n} \Omega_{n}$ for $C_{*}$, and we write $\left(C_{*}, \Omega\right)$ to denote a chain complex with a basis. A free chain complex with a basis is the main character of this paper.

Given a free chain complex with a basis $\left(C_{*}, \Omega\right)$, and two elements $\alpha \in C_{n}$, and $b \in \Omega_{n}$, we denote the coefficient of $b$ in the representation of $\alpha$ as a linear combination of the elements of $\Omega_{n}$ by $\mathfrak{K}_{\Omega}(\alpha, b)$, or, if the basis is clear, simply by $\mathfrak{K}(\alpha, b)$. For $x \in C_{n}$ we write $\operatorname{dim} x=n$. By convention, we set $\mathfrak{K}_{\Omega}(\alpha, b)=0$ if the dimensions do not match, i.e., if $\operatorname{dim} \alpha \neq \operatorname{dim} b$.

Note that a free chain complex with a basis $\left(C_{*}, \Omega\right)$ can be represented as a ranked poset $P\left(C_{*}, \Omega\right)$, with $\mathcal{R}$-weights on the order relations. The elements of rank $n$ correspond to the elements of $\Omega_{n}$, and the weight of the covering relation $b \succ a$, for $b \in \Omega_{n}, a \in \Omega_{n-1}$, is simply defined by $w_{\Omega}(b \succ a):=\mathfrak{K}_{\Omega}(\partial b, a)$. In other words, $\partial b=\sum_{b \succ a} w_{\Omega}(b \succ a) a$, for each $b \in \Omega_{n}$. Again, if the basis is clear, we simply write $w(b \succ a)$.

Definition 1.1 Let $\left(C_{*}, \Omega\right)$ be a free chain complex with a basis. A partial matching $\mathcal{M} \subseteq \Omega \times \Omega$ on $\left(C_{*}, \Omega\right)$ is a partial matching on the covering graph of $P\left(C_{*}, \Omega\right)$, such that if $b \succ a$, and $b$ and $a$ are matched, i.e. if $(a, b) \in M$, then $w(b \succ a)$ is invertible.

Remark 1 Note that the Definition 1.1 is different from [1, Definition 2.1]. The latter is a topological definition, and has the condition that the matched cells form a regular pair (in the $C W$ sense). In our algebraic setting it suffices to require the invertibility of the covering weight.

Given such a partial matching $\mathcal{M}$, we write $b=u(a)$, and $a=d(b)$, if $(a, b) \in \mathcal{M}$. We denote by $\mathcal{U}_{n}(\Omega)$ the set of all $b \in \Omega_{n}$, such that $b$ is matched with some $a \in \Omega_{n-1}$, and, analogously, we denote by $\mathcal{D}_{n}(\Omega)$ the set of all $a \in \Omega_{n}$, which are matched with some $b \in \Omega_{n+1}$. We set $\mathcal{C}_{n}(\Omega):=\Omega_{n} \backslash\left\{\mathcal{U}_{n}(\Omega) \cup \mathcal{D}_{n}(\Omega)\right\}$ to be the set of all unmatched basis elements; these elements are called critical. Finally, we set $\mathcal{U}(\Omega):=\bigcup_{n} \mathcal{U}_{n}(\Omega)$, $\mathcal{D}(\Omega):=\bigcup_{n} \mathcal{D}_{n}(\Omega)$, and $\mathcal{C}(\Omega):=\bigcup_{n} \mathcal{C}_{n}(\Omega)$.

Given two basis elements $s \in \Omega_{n}$ and $t \in \Omega_{n-1}$, an alternating path is a sequence

$$
p=\left(s \succ d\left(b_{1}\right) \prec b_{1} \succ d\left(b_{2}\right) \prec b_{2} \succ \ldots \succ d\left(b_{n}\right) \prec b_{n} \succ t\right),
$$

where $n \geq 0$, and all $b_{i} \in \mathcal{U}(\Omega)$ are distinct. We use the notations $p^{\bullet}=s$ and $p_{\bullet}=t$. The weight of such an alternating path is defined to be the quotient

$$
w(p):=(-1)^{n} \frac{w\left(s \succ d\left(b_{1}\right)\right) \cdot w\left(b_{1} \succ d\left(b_{2}\right)\right) \cdot \ldots \cdot w\left(b_{n} \succ t\right)}{w\left(b_{1} \succ d\left(b_{1}\right)\right) \cdot w\left(b_{2} \succ d\left(b_{2}\right)\right) \cdot \ldots \cdot w\left(b_{n} \succ d\left(b_{n}\right)\right)} .
$$

Definition 1.2 A partial matching on $\left(C_{*}, \Omega\right)$ is called acyclic, if there does not exist a cycle

$$
d\left(b_{1}\right) \prec b_{1} \succ d\left(b_{2}\right) \prec b_{2} \succ d\left(b_{3}\right) \prec \ldots \succ d\left(b_{n}\right) \prec b_{n} \succ d\left(b_{1}\right),
$$

with $n \geq 2$, and all $b_{i} \in \mathcal{U}(\Omega)$ being distinct.

There is a nice alternative way to reformulate the notion of acyclic matching.

Proposition 1.3 A partial matching on $\left(C_{*}, \Omega\right)$ is acyclic if and only if there exists a linear extension of $P\left(C_{*}, \Omega\right)$, such that, in this extension $u(a)$ follows directly after a, for all $a \in \mathcal{D}(\Omega)$.

This extension can always be chosen so that, restricted to $\mathcal{D}(\Omega) \cup \mathcal{C}(\Omega)$, it does not decrease rank.

Proof. If such an extension $L$ exists, then following a cycle (3) left to right we always go down in the order $L$ (more precisely, moving one position up is followed by moving at least two positions down), hence a contradiction.

Assume that the matching is acyclic, and define $L$ inductively. Let $Q$ denote the set of elements which are already ordered in $L$. We start with $Q=\emptyset$. Let $W$ denote the set of the lowest rank elements in $P\left(C_{*}, \Omega\right) \backslash Q$. At each step we have one of the following cases.

Case 1. One of the elements $c$ in $W$ is critical. Then simply add $c$ to the order $L$ as the largest element, and proceed with $Q \cup\{c\}$. 
Case 2. All elements in $W$ are matched. The covering graph induced by $W \cup u(W)$ is acyclic, hence the total number of edges is at most $2|W|-1$. It follows that there exists $a \in W$, such that $P\left(C_{*}, \Omega\right)_{<u(a)} \backslash Q=\{a\}$. Hence, we can add elements $a$ and $u(a)$ on top of $L$ and proceed with $Q \cup\{a, u(a)\}$.

Definition 1.4 Let $\left(C_{*}, \Omega\right)$ be a free chain complex with a basis, and let $\mathcal{M}$ be an acyclic matching. The Morse complex ... $\stackrel{\partial_{n+2}^{\mathcal{M}}}{\longrightarrow} C_{n+1}^{\mathcal{M}} \stackrel{\partial_{n+1}^{\mathcal{M}}}{\longrightarrow} C_{n}^{\mathcal{M}} \stackrel{\partial_{n}^{\mathcal{M}}}{\longrightarrow} C_{n-1}^{\mathcal{M}} \stackrel{\partial_{n-1}^{\mathcal{M}}}{\longrightarrow} \ldots$, is defined as follows. The $\mathcal{R}$-module $C_{n}^{\mathcal{M}}$ is freely generated by the elements of $\mathcal{C}_{n}(\Omega)$. The boundary operator is defined by $\partial_{n}^{\mathcal{M}}(s)=\sum_{p} w(p) \cdot p_{\bullet}$, for $s \in \mathcal{C}_{n}(\Omega)$, where the sum is taken over all alternating paths $p$ satisfying $p^{\bullet}=s$.

Again, if the indexing is clear, we simply write $\partial^{\mathcal{M}}$ instead of $\partial_{n}^{\mathcal{M}}$.

Given a free chain complex with a basis $\left(C_{*}, \Omega\right)$, we can choose a different basis $\widetilde{\Omega}$ by replacing each $a \in \mathcal{D}_{n}(\Omega)$ by $\tilde{a}=w(u(a) \succ a) \cdot a$. Since

$$
\mathfrak{K}_{\widetilde{\Omega}}(x, \tilde{a})=\mathfrak{K}_{\Omega}(x, a) / w(u(a) \succ a),
$$

for any $x \in \Omega_{n}$, we see that the weights of those alternating paths, which do not begin with or end in an element from $\mathcal{D}_{n}(\Omega)$, remain unaltered, as the quotient $w(x \succ z) / w(y \succ z)$ stays constant as long as $x, y \neq a$. In particular, the Morse complex will not change. On the other hand, by $(4), w_{\widetilde{\Omega}}(u(a) \succ a)=1$, for all $a \in \mathcal{D}(\widetilde{\Omega})$, so the total weight of the alternating path in (2) will simply become

$$
w_{\widetilde{\Omega}}(p)=(-1)^{n} w_{\widetilde{\Omega}}\left(s \succ d\left(b_{1}\right)\right) \cdot w_{\widetilde{\Omega}}\left(b_{1} \succ d\left(b_{2}\right)\right) \cdot \ldots \cdot w_{\widetilde{\Omega}}\left(b_{n} \succ t\right) .
$$

Because of these observations, we may always replace any given basis of $C_{*}$ with the basis $\widetilde{\Omega}$ satisfying $w_{\widetilde{\Omega}}(u(a) \succ a)=1$, for all $a \in \mathcal{D}(\widetilde{\Omega})$.

\section{The main theorem.}

The chain complex $\ldots \longrightarrow 0 \longrightarrow \mathcal{R} \stackrel{\mathrm{id}}{\longrightarrow} \mathcal{R} \longrightarrow 0 \longrightarrow \ldots$, where the only nontrivial modules are in the dimensions $d$ and $d-1$, is called an atom chain complex, and is denoted by Atom $(d)$.

The main result brings to light a certain structure in $C_{*}$. Namely, by choosing a different basis, we will represent $C_{*}$ as a direct sum of two chain complexes, of which one is a direct sum of atom chain complexes, in particular acyclic, and the other one is isomorphic to $C_{*}^{\mathcal{M}}$. For convenience, the choice of basis will be performed in several steps, one step for each matched pair of the basis elements.

Theorem 2.1 Assume that we have a free chain complex with a basis $\left(C_{*}, \Omega\right)$, and an acyclic matching $\mathcal{M}$. Then $C_{*}$ decomposes as a direct sum of chain complexes $C_{*}^{\mathcal{M}} \oplus T_{*}$, where $T_{*} \simeq \bigoplus_{(a, b) \in \mathcal{M}} \operatorname{Atom}(\operatorname{dim} b)$. Proof. To start with, let us choose a linear extension $L$ of the partially ordered set $P\left(C_{*}, \Omega\right)$ satisfying the conditions of the Proposition 1.3, and let $<_{L}$ denote the corresponding total order.

Assume first that $C_{*}$ is bounded; without loss of generality, we can assume that $C_{i}=0$ for $i<0$, and $i>N$. Let $m=|M|$ denote the size of the matching, and let $l=|\Omega|-2 m$ denote the number of critical cells.

We shall now inductively construct a sequence of bases $\Omega^{0}, \Omega^{1}, \ldots, \Omega^{m}$ of $C_{*}$. Finer, each basis will be divided into three parts: $\mathcal{C}\left(\Omega^{k}\right)=\left\{c_{1}^{k}, \ldots, c_{l}^{k}\right\}, \mathcal{D}\left(\Omega^{k}\right)=\left\{a_{1}^{k}, \ldots, a_{m}^{k}\right\}$, and $\mathcal{U}\left(\Omega^{k}\right)=\left\{b_{1}^{k}, \ldots, b_{m}^{k}\right\}$, such that $a_{i}^{k}=d\left(b_{i}^{k}\right)$, for all $i \in[m]$.

We start with $\Omega^{0}=\Omega$ and the initial condition $b_{i}^{0}<_{L} b_{i+1}^{0}$, for all $i \in[m-1]$. Since the lower index of $\mathfrak{K}_{-}(-,-)$and $w_{-}(-\succ-)$ will be clear from the arguments, we shall omit it to make the formulae more compact.

When constructing the bases, we shall simultaneously prove by induction the following statements: (i) $C_{*}=C_{*}[k] \oplus \mathcal{A}_{1}^{k} \oplus \ldots \oplus \mathcal{A}_{k}^{k}$, where $C_{*}[k]$ is the subcomplex of $C_{*}$ generated by $\Omega^{k} \backslash$ $\left\{a_{1}^{k}, \ldots, a_{k}^{k}, b_{1}^{k}, \ldots, b_{k}^{k}\right\}$, and $\mathcal{A}_{i}^{k}$ is isomorphic to $\operatorname{Atom}\left(\operatorname{dim} b_{i}^{k}\right)$, for $i \in[k]$; 
(ii) for every $x^{k} \in \mathcal{U}\left(\Omega^{k}\right) \cup \mathcal{C}\left(\Omega^{k}\right), y \in \mathcal{C}\left(\Omega^{k}\right)$, we have $w\left(x^{k} \succ y^{k}\right)=\sum_{p} w(p)$, where the sum is restricted to those alternating paths from $x^{0}$ to $y^{0}$ which only use the pairs $\left(a_{i}^{0}, b_{i}^{0}\right)$, for $i \in[k]$.

Clearly, all of the statements are true for $k=0$. Assume $k \geq 1$.

Transformation of the basis $\Omega^{k-1}$ into the basis $\Omega^{k}$ : set $a_{k}^{k}:=\partial b_{k}^{k-1}, b_{k}^{k}:=b_{k}^{k-1}$, and $x^{k}:=x^{k-1}-$ $w\left(x^{k-1} \succ a_{k}^{k-1}\right) \cdot b_{k}^{k-1}$, for all $x^{k-1} \in \Omega^{k-1}, x \neq a_{k}, b_{k}$.

First, we see that $\Omega^{k}$ is a basis. Indeed, assume $b_{k}^{k-1} \in C_{n}$. For $i \neq n, n-1$, we have $\Omega_{i}^{k}=\Omega_{i}^{k-1}$, hence, by induction, it is a basis. $\Omega_{n-1}^{k}$ is obtained from $\Omega_{n-1}^{k-1}$ by adding a linear combination of other basis elements to the basis element $a_{k}^{k-1}$, hence $\Omega_{n-1}^{k}$ is again a basis. Finally, $\Omega_{n}^{k}$ is obtained from $\Omega_{n}^{k-1}$ by subtracting multiples of the basis element $b_{k}^{k-1}$ from the other basis elements, hence it is also a basis.

Next, we investigate how the poset $P\left(C_{*}, \Omega^{k}\right)$ differs from $P\left(C_{*}, \Omega^{k-1}\right)$. If $x \neq b_{k}$, we have $w\left(x^{k} \succ\right.$ $\left.a_{k}^{k}\right)=\mathfrak{K}\left(\partial x^{k}, a_{k}^{k}\right)=\mathfrak{K}\left(\partial x^{k}, a_{k}^{k-1}\right)=\mathfrak{K}\left(\partial x^{k-1}, a_{k}^{k-1}\right)-w\left(x^{k-1} \succ a_{k}^{k-1}\right) \cdot \mathfrak{K}\left(\partial b_{k}^{k-1}, a_{k}^{k-1}\right)=0$, where the second equality follows from the fact that $\Omega_{n-1}^{k}$ is obtained from $\Omega_{n-1}^{k-1}$ by adding a linear combination of other basis elements to the basis element $a_{k}^{k-1}$, and the last equality follows from $\mathfrak{K}\left(\partial b_{k}^{k-1}, a_{k}^{k-1}\right)=1$.

Furthermore, since $\Omega_{n}^{k}$ is obtained from $\Omega_{n}^{k-1}$ by subtracting multiples of the basis element $b_{k}^{k-1}$ from the other basis elements, we see that for $x \in \Omega_{n+1}^{k}, y \in \Omega_{n}^{k}, y \neq b_{k}$, we have $w\left(x^{k} \succ y^{k}\right)=w\left(x^{k-1} \succ\right.$ $\left.y^{k-1}\right)$. Additionally, since the differential of the chain complex squares to 0 , we have $0=\sum_{z^{k} \in \Omega_{n}^{k}} w\left(x^{k} \succ\right.$ $\left.z^{k}\right) \cdot w\left(z^{k} \succ a_{k}^{k}\right)=w\left(x^{k} \succ b_{k}^{k}\right) \cdot w\left(b_{k}^{k} \succ a_{k}^{k}\right)=w\left(x^{k} \succ b_{k}^{k}\right)$, where the second equality follows from $w\left(z^{k} \succ a_{k}^{k}\right)=0$, for $z \neq b_{k}$.

We can summarize our findings as follows: all weights in the poset $P\left(C_{*}, \Omega^{k}\right)$ are the same as in $P\left(C_{*}, \Omega^{k-1}\right)$, with the following exceptions:

1) $w\left(x^{k} \succ b_{k}^{k}\right)=0$, and $w\left(b_{k}^{k} \succ x^{k}\right)=0$, for $x \neq a_{k}$;

2) $w\left(a_{k}^{k} \succ x^{k}\right)=0$, and $w\left(x^{k} \succ a_{k}^{k}\right)=0$, for $x \neq b_{k}$;

3) $w\left(x^{k} \succ y^{k}\right)=w\left(x^{k-1} \succ y^{k-1}\right)-w\left(x^{k-1} \succ a_{k}^{k-1}\right) \cdot w\left(b_{k}^{k-1} \succ y^{k-1}\right)$, for $x \in \Omega_{n}^{k}, y \in \Omega_{n-1}^{k}, x \neq b_{k}$, $y \neq a_{k}$.

In particular, the statement $(i)$ is proved. Furthermore, the following fact $(*)$ can be seen by induction, using 1), 2), and 3): if $w\left(x^{k} \succ y^{k}\right) \neq w\left(x^{k-1} \succ y^{k-1}\right)$, then $b_{k}^{0} \geq_{L} y^{0}$. Indeed, either $y \in\left\{a_{k}, b_{k}\right\}$, or $y$ is critical, or $y=a_{\tilde{k}}$, for $\tilde{k}>k$, such that $w\left(b_{k}^{k-1} \succ y^{k-1}\right) \neq 0$. In the first two cases $b_{k}^{0} \geq_{L} y^{0}$ by the construction of $L$, and the last case is impossible by induction, and again, by the construction of $L$.

We have $w\left(b_{j}^{k} \succ a_{j}^{k}\right)=w\left(b_{j}^{k-1} \succ a_{j}^{k-1}\right)$, for all $j, k$. Indeed, this is clear for $j=k$. The case $j<k$ follows by induction, and the case $j>k$ is a consequence of the fact $(*)$.

Next, we see that the partial matching $\mathcal{M}^{k}:=\left\{\left(a_{i}^{k}, b_{i}^{k}\right) \mid i \in[m]\right\}$ is acyclic. For $j \leq k$, the poset elements $b_{j}^{k}, a_{j}^{k}$ are incomparable with the rest, hence they cannot be a part of a cycle. For $i>k$, we have $w\left(b_{j}^{k} \succ a_{i}^{k}\right)=w\left(b_{j}^{k-1} \succ a_{i}^{k-1}\right)$, by the fact $(*)$. Hence, by induction, no cycle can be formed by these elements either.

Finally, we trace the boundary operator. Let $x^{k} \in \mathcal{U}\left(\Omega^{k}\right) \cup \mathcal{C}\left(\Omega^{k}\right), y \in \mathcal{C}\left(\Omega^{k}\right)$. For $x=b_{k}$ the statement is clear. If $x \neq b_{k}$, we have $w\left(x^{k} \succ y^{k}\right)=w\left(x^{k-1} \succ y^{k-1}\right)-w\left(x^{k-1} \succ a_{k}^{k-1}\right) w\left(b_{k}^{k-1} \succ y^{k-1}\right)$. By induction, the first term is counting the contribution of all the alternating paths from $x^{0}$ to $y^{0}$ which do not use the edges $b_{l}^{0} \succ a_{l}^{0}$, for $l \geq k$. The second term contains the additional contribution of the alternating paths from $x^{0}$ to $y^{0}$ which use the edge $b_{k}^{0} \succ a_{k}^{0}$. Observe, that if this edge occurs then, by the construction of $L$, it must be the second edge of the path (counting from $x^{0}$ ), and, by the fact $(*)$, we have $w\left(x^{k-1} \succ a_{k}^{k-1}\right)=w\left(x^{0} \succ a_{k}^{0}\right)$. This proves the statement $(i i)$, and therefore concludes the proof of the finite case.

It is now easy to deal with the infinite case, since the basis stabilizes as we proceed through the dimensions, so we may take the union of the stable parts as the new basis for $C_{*}$.

Remark 2 Even if the chain complex $C^{*}$ is infinite in both directions, one can still define the notion of 
the acyclic matching and of the Morse complex. Since each particular homology group is determined by a finite excerpt from $C^{*}$, we may still conclude that $H_{*}\left(C_{*}\right)=H_{*}\left(C_{*}^{\mathcal{M}}\right)$.

\section{References}

[1] R. Forman, Morse theory for cell complexes, Adv. Math. 134, (1998), no. 1, 90-145.

[2] D.N. Kozlov, Collapsibility of $\Delta\left(\Pi_{n}\right) / \mathcal{D}_{n}$ and some related $C W$ complexes, Proc. Amer. Math. Soc. 128, (2000), no. 8, 2253-2259. 